\title{
Linx
}

Revue des linguistes de l'université Paris X Nanterre

40 | 1999

Le statut d'unité lexicale

\section{Une ballade en toponymie : de la rue Descartes à la rue de Rennes}

A toponymic walk from rue Descartes to rue de Rennes.

\section{Bernard Bosredon et Irène Tamba}

\section{(2) OpenEdition}

Journals

\section{Édition électronique}

URL : http://journals.openedition.org/linx/743

DOI : $10.4000 / \operatorname{linx} .743$

ISSN : 2118-9692

\section{Éditeur}

Presses universitaires de Paris Nanterre

\section{Édition imprimée}

Date de publication : 1 juin 1999

Pagination : 55-69

ISSN : 0246-8743

\section{Référence électronique}

Bernard Bosredon et Irène Tamba, « Une ballade en toponymie : de la rue Descartes à la rue de

Rennes », Linx [En ligne], 40 | 1999, mis en ligne le 20 juin 2012, consulté le 20 avril 2019. URL : http:// journals.openedition.org/linx/743; DOI : 10.4000/linx.743

Ce document a été généré automatiquement le 20 avril 2019.

Département de Sciences du langage, Université Paris Ouest 


\section{Une ballade en toponymie : de la rue Descartes à la rue de Rennes}

A toponymic walk from rue Descartes to rue de Rennes.

Bernard Bosredon et Irène Tamba

« L'appellation des rues est un sujet extrêmement complexe, et souvent même épineux, à propos duquel on pourrait écrire plusieurs ouvrages ». G. Perec, Espèces d'espaces, p. 65-66, Galilée, Paris.

\section{Introduction}

1 Les noms de rue ont été jusqu'ici étudiés principalement par les historiens et les spécialistes d'onomastique. Un "beau sujet" pour les premiers qui ne pouvaient, comme l'écrit D. Milo, « résister aux charmes des chaînes dénominatives ", source inépuisable de plaisanteries et d'anecdotes colorées ${ }^{1}$. Une vraie caverne d'Ali Baba pour les seconds qui sont évidemment chez eux dans l'analyse d'appellations proliférantes. Cependant, entre la recherche des déterminations historiques et la description fine des sédimentations philologiques de cet ensemble particulier de noms de lieux, il y a place pour une approche spécifiquement linguistique. Bien qu'à peine explorée, cette voie nous paraît seule capable de donner accès aux principes sémantico-syntaxiques qui régulent la construction de ces dénominations complexes.

2 Partant donc de l'hypothèse que la facture des dénominations complexes propres aux voies de communication doit ses schémas récurrents à ce que l'un de nous a appelé une signalétique (Bosredon, 1997 : 285-315), c'est-à-dire une activité de nomination d'objets uniques soumise à des contraintes pragmatiques locales, nous nous proposons d'examiner l'organisation signalétique dont dépend en français la nomination des voies de communication ou odonymes. A cet effet, nous commencerons par dégager les caractéristiques générales des signalétiques propres aux odonymes routiers d'une part et urbains de l'autre. Puis, nous examinerons plus en détail les odonymes urbains formés d'un premier nom classificateur (désormais $\mathrm{N}_{\mathrm{c}}$ ), tel avenue, rue, etc. et d'un deuxième 
nom propre individualisateur(désormais $\mathrm{N} 2$ pr ). Nous montrerons que ces odonymes font appel à deux modes de couplage distincts : soit par juxtaxposition, si $\mathrm{N}_{\mathrm{pr}}$ est un nom de personne : rue Gambetta; soit par jonction à l'aide de la préposition de, si $\mathrm{N}_{\mathrm{pr}}$ est un nom de lieu rue de Metz... C'est l'énigme que pose cette bipartition structurelle que le présent article tentera de résoudre.

\section{La dénomination des voies de communication}

\subsection{Une première dichotomie : odonymie routière, odonymie urbaine}

3 En France aujourd'hui, les voies de communication relèvent de deux organisations onomastiques différentes, selon qu'il s'agit du réseau routier ou du réseau urbain. La nomenclature des routes est régie par un code taxinomique homogène et stable, alors que celle des voies urbaines dépend de l'amalgame de plusieurs systèmes mis en place par strates successives au cours de l'histoire.

4 Les noms de route sont formés suivant un patron binaire régulier couplant une catégorie institutionnelle et un chiffre : nationale 6, départementale 12, le plus souvent abrégés en N6, D12. Le premier élément correspond à un nom féminin issu de la condensation d'un syntagme, composé de route et d'un adjectif: route nationale. On a donc affaire à une combinatoire alphanumérique à deux places, qui génère des unités syntagmatiques dont les deux membres ne sont unis par aucun lien syntaxique. Tous les noms de routes sont formés sur ce modèle, et seules quelques autoroutes reçoivent de surcroît une dénomination particulière, comme l'A11, dite l'Océane.

5 La formation des odonymes urbains repose, elle aussi, sur un principe de couplage binaire, conjoignant un premier élément nominal classificateur à un deuxième élément individualisateur. Mais là s'arrête la ressemblance. Car elle fait appel à des ensembles différents de termes classificateurs et individualisateurs ainsi qu'à deux modes de jonction internominale. On répertorie en effet une vingtaine de classificateurs urbains ${ }^{3}$, tels que rue, boulevard, place, pont, etc., qui ne découlent pas d'un système taxinomique doté d'une logique classificatoire uniforme, mais de l'accumulation d'appellations catégorielles, ajoutés au fil des époques. Comme le rappelle Rastier (1991:60), «rue est attesté en 1080, avenue en 1549, cours en 1616, boulevard en $1803 \%$. Par ailleurs rue s'emploie aujourd'hui tantôt comme terme générique : un plan de Paris comporte une "nomenclature des rues de Paris", tantôt comme premier élément nominal classificateur, désignant en principe une voie moins large que boulevard. On relève également des classificateurs comme carré (carré des Champs-Elysées), pont (pont Alexandre-III), villa (villa Adrienne), en plus petit nombre, qui évoquent moins naturellement que les précédents l'idée d'une "voie". Nous considérerons que toutes les formes N1 qui entrent dans une nomenclature de noms de rues ( $C f$. note $\left.{ }^{5}\right)$ sont à ranger dans les noms de rues et qu'un corpus comme celui que contient le plan Paris Eclair de Leconte ( $C$. note 5), corpus où nous puiserons nos exemples, sera à la fois suffisant et consistant, même si une analyse plus fine pourrait révéler des spécificités dans le choix des N2 que certains N1 sélectionnent (place ou pont, par ex.).

6 De leur côté les GN2 des odonymes urbains présentent une certaine variété, par rapport à ceux, exclusivement numériques, des odonymes routiers ${ }^{5}$. On exploite différents traits de 
caractérisation individualisante : particularités contingentes (rue des Quatre fils), direction (avenue de Versailles), commémoration de lieux, dates et personnages historiques ou de vertus (quai d'Austerlitz, place Napoléon III, rue du 14-Juillet, rue de la Liberté) ou encore séries thématiques : arbres, fleurs, oiseaux, points cardinaux, etc., (rue des Ormeaux, rue de l'Ouest ).

7 Enfin la jonction des deux segments relève soit d'une construction syntaxique soit d'une combinatoire extra-linguistique qui se projette syntagmatiquement dans la langue sous la forme d'une simple séquentialisation. Dans le premier cas, on trouve des constructions adjectivales (rue Longue, Grand-Rue) et des groupes prépositionnels presque exclusivement en de (rue du Chat-qui-pêche) ${ }^{6}$. Dans le second cas, le nom catégorisateur est directement suivi d'un nom propre, simple ou complexe (rue Rabelais, rue Saint-Vincent-de-Paul).

\subsection{Propriétés sémiotiques de ces dénominations complexes d'objets uniques (DCOU)}

Du point de vue sémiotique les odonymes, bien que de facture complexe, fonctionnent comme une unité compacte et fixe dénommant un objet unique par commune. Ils répondent ainsi aux questions d'identification appellative :

- Comment s'appelle, quel est le nom de cette route/de cette rue?

10 - C'est la N7 / la rue du Télégraphe

11 Il est clair que le Npr ne peut servir seul à nommer la rue. On ne dira pas: *cette rue s'appelle Bonaparte, mais bien: cette rue s'appelle rue Bonaparte. C'est donc l'ensemble $\mathrm{N}_{\mathrm{c}}$ $+\mathrm{N} 2_{\mathrm{pr}}$ qui forme le nom de la voie.

12 Plusieurs observations confirment ce statut appellatif. La forme bisegmentale est inaltérable. A l'exception de quelques voies célèbres, qui ont acquis une identité autonome, comme la Canebière ou les Champs Elysées, on ne peut faire l'économie du formant catégoriel, partie intégrante de la dénomination. Et même des formes abrégées, telles que Boul'Mich ou N7, préservent normalement la facture bisegmentale de base ${ }^{7}$.

13 L'orthographe par ailleurs impose des normes graphiques qui accentuent la structure bisegmentale et l'homogénéisation de ces DCOUs. Comme l'a bien vu K. Togeby (1982: $156)^{8}$, il est d'usage de souder les éléments d'un second segment complexe par des traits d'union et il cite l'exemple de la rue des Fossés-Saint-Germain-des-Prés. D'autre part, le nom catégorisateur de tête s'écrit avec une minuscule initiale, mais chaque constituant du segment individuant commence par une majuscule. Ainsi sépare-t-on graphiquement les deux constituants des odonymes et marque-t-on le rôle identificateur du second segment en lui conférant la majuscule caractéristique des noms propres d'individus, par opposition au rôle catégorisateur du premier nom qui reçoit la minuscule des noms communs.

\subsection{Propriétés sémantiques}

Du point de vue sémantique, les odonymes ont un statut, à première vue, paradoxal. Car si d'un côté on a bien affaire à des appellations sémantiquement analysables en deux composants qui ont un sens - éventuellement traduisible - d'un autre côté leur fonctionnement d'identificateur nominal rigide semble indépendant de leur signification intrinsèque. Les catégories constituent certes des cases de rangement en nombre limité 
mais n'instaurent pas de véritable hiérarchie classificatoire, définie à l'aide de critères systématiques homogènes. N'a-t-on pas affaire plutôt à une nomenclature réunissant plusieurs systèmes de répartition, historiquement et géographiquement stratifiés? Les données historiques corroborent cette hypothèse. Les odonymes sont en effet fixés par un acte de baptême communautaire ou institutionnel, souvent réitéré, selon les vicissitudes de l'histoire. Une série de prédicats permet d'exprimer ces aléas de la nomination : on débaptise une rue, on dit qu'une rue a changé de nom, qu'elle a pris le nom de, qu'elle était anciennement nommée, etc. Et, comme le montre D. Milo (1986 : 285-288), en France la nomenclature des rues est passée « de l'initiative populaire au monopole étatique ». Le système médiéval repose sur "une mémoire populaire" attachée aux caractéristiques des lieux (rue de l'Horloge). La nomenclature institutionnelle, au contraire, met en place "un système honorifique", dont la paternité est attribuée à Sully, qui, au début du 17e siècle, aurait eu l'idée « d'honorer les grands du royaume en inscrivant leurs noms sur le plan de Paris » (ibid.). La nomination des rues est dès lors fondée sur une mémoire officielle, commémorant les héros et les événements politiques et militaires.

La nomination des rues répond donc à une double postulation sémantique: celle d'identifier des rues par une appellation propre et celle de participer à l'élaboration d'une mémoire collective populaire ou officielle, à une échelle locale ou nationale.

\subsection{Caractéristiques pragmatiques}

Les signalétiques répondent à un double besoin : celui de délimiter une catégorie et celui de permettre l'identification de tout objet particulier qu'on veut y rattacher. Ce sont donc certains usages qui, dans des champs d'activité déterminés, réclament une signalétique.

Dans le cas du réseau routier, c'est la nécessité de guider des déplacements à distance plus ou moins longue qui a joué un rôle déterminant, avec l'instauration de panneaux de signalisation qui jalonnent un itinéraire. La carte routière, de son côté, impose une nomenclature simple et stable de type alphanumérique, munie d'un système propre de décodage sous la forme de légendes explicatives. La compacité, la simplicité, la construction régulière des noms de cette nomenclature est soumise à des conventions aussi strictes que celles qui règlent, par ailleurs, la représentation cartographique de l'espace routier. Cet usage officiel n'exclut pas toutefois le recours à des indications officieuses, moins élaborées, qui relèvent d'une orientation topographique vécue et sont de règle notamment à la campagne. On parlera alors de direction, de lieu de départ et d'arrivée ou on emploiera des désignations locales en disant par exemple: prendre vers l'Ouest "la route de Bordeaux à Arcachon" ou se diriger vers le Nord-Ouest en cherchant "la route des vins" ou "la route des châteaux"; ou encore, suivre au Nord la "route des berges", bifurquer vers "la route des côteaux" et suivre ensuite "la route des crêtes"....

En ce qui concerne les autoroutes, on note que la nomenclature cartographique est relativement récente et, de toute façon, bien postérieure à la terminologie de l'odonymie traditionnelle. Mais si elle suit les règles générales de l'odonymie routière ( $A 1, A 62$ etc.), elle ne semble pas enregistrer, sur les cartes, les appellations courantes du type de celle que nous citions plus haut (l'océane). L'autoroute du Nord, l'autoroute des deux mers, l'autoroute du soleil, etc. appartiennent au langage courant et sont parfois signalées entre parenthèses sur les cartes routières. Bref, à côté de la nomenclature cartographique construite sur un modèle alphanumérique régulier existent des désignations stables constituant une signalétique différente. Cette variation du type de dénomination est, on 
le voit pragmatiquement conditionnée 9 . On considérera en effet que ces noms, qui ne ressortissent pas de la nomenclature cartographique, se comportent comme des surnoms doublant en quelque sorte une nomenclature abstraite et conférant, à l'inverse, aux dénominations ainsi construites une capacité communicative plus grande, dont la source est plus diffuse et certainement moins intentionnelle.

La dénomination des rues diffère de celle des noms des routes, car elle ne remplit pas les mêmes fonctions. Sans doute les rues servent-elles comme les routes à circuler - dans un espace urbain bien particulier toutefois - mais elles jouent aussi un rôle capital dans le découpage de la ville et le repérage des habitations. Par ailleurs, la poste impose un système d'adresses, avec mention des noms de rue et des numéros des immeubles, qui contribue fortement à fixer le nom exact des rues. G. Perec, en fin observateur, a bien relevé ces caractéristiques :

«L'alignement parallèle de deux séries d'immeubles détermine ce que l'on appelle une rue : la rue est un espace bordé, généralement sur ses deux plus longs côtés, de maisons; la rue est ce qui sépare les maisons les unes des autres, et aussi ce qui permet d'aller d'une maison à d'autre, soit en longeant, soit en traversant la rue. De plus, la rue est ce qui permet de repérer les maisons. Il existe différents systèmes de repérage ; le plus répandu, de nos jours et sous nos climats, consiste à donner un nom à la rue et des numéros aux maisons. » (Perec, 1974 : 65).

21 La signalisation des rues se fait au moyen de plaques apposées à leurs extrémités et à leurs croisements. Ainsi se constitue un réseau urbain que figure un plan dont la consultation se fait à l'aide d'un découpage administratif (en arrondissements, pour Paris) et d'un index alphabétique. On le voit, un plan urbain sert essentiellement à localiser des voies et à trouver des adresses, alors qu'une carte routière aide avant tout à visualiser des trajets sur de grandes distances, sans oublier que ces deux systèmes coexistent avec divers modes de repérage officieux des lieux familiers.

\subsection{Bilan provisoire}

Ce rapide examen a mis en évidence quatre points essentiels :

23 (1) La relative hétérogénéité sémiotique des voies urbaines face à l'homogénéité sémiotique de la nomenclature des voies routières.

(2) Pour la nomenclature des routes, l'existence d'une organisation sémiotique présentant une régularité optimale : un seul schéma de couplage par séquentialisation ordonnée ; un nombre minimal de catégories, correspondant à un ensemble hiérarchique de divisions juridiques administratives : canton, département, état. Seul le réseau autoroutier, qui relève de sociétés privées, n'est que partiellement intégré à cet ordre sémiotique, avec une appellation généralement conforme à la facture des noms de route sur les cartes routières standard (A4) et une autre plus géographique (la Francilienne, l'Autoroute du Soleil, etc.).

25 (3) Pour la nomenclature des rues, la coexistence de deux organisations antagonistes: l'une, sémiotique, tend comme dans le cas des routes à imposer une facture bisegmentale homogène à premier élément catégorisateur et à second élément différenciateur ; l'autre, sémantique, soumise à des déterminations politico-historiques, dérange l'ordre sémiotique, en introduisant au fil du temps des catégories sans lien systématique entre elles (sentier, allée, boulevard), et en recourant à des éléments différenciateurs hétérogènes, selon les besoins ou l'idéologie politique du moment. 
26 (4) L'opposition entre l'unique schéma de couplage, par juxtaposition ordonnée, des deux segments constitutifs des noms de route, et le double schéma de liaison des deux parties des noms de rue : soit syntaxique, au moyen de la préposition de; soit paratactique, par juxtaposition directe, comme dans le cas des noms de route.

27 A quoi tient la coexistence de ces deux patrons syntaxiques? C'est ce que nous allons essayer d'élucider à présent, en analysant de plus près un sous-groupe de noms de rue dont la répartition entre ces deux schèmes de couplage est au premier abord des plus aléatoire. Il s'agit des noms de rue dont l'élément individualisateur comporte un nom propre.

\section{Double structure des noms de rue dont le N2 est un nom propre}

\subsection{Présentation des structures à $\mathrm{N} 2$ pr.}

On dispose de deux schèmes réguliers pour construire les noms de rue, qu'illustrent les exemples cités dans le titre du présent article :

(1) rue Descartes

(2) rue de Rennes

Comme le rappelle L. Palm (1989: 9), K. Togeby impute cette différence de construction au type de nom propre employé. La préposition intérieure de suivie ou non d'un article défini, précise-t-il, se trouve devant les "noms de villes" (rue du Havre), "les noms de fleuves" (rue du Loiret) devant "les noms de pays" qui «entrent assez rarement dans les noms des rues » comme par exemple rue d'Argentine. Et d'ajouter :

«Devant les noms de personne, on évite en général la préposition de : boulevard Voltaire (...). Dans les rares cas où on a de, il peut s'agir de la particule nobiliaire : rue de Condé (...). Si le nom de personne est précédé d'un titre, le de devient nécessaire (...) : rue de l'Abbé-de-l'Epée. Saint n'est pas un titre, d'où l'absence de la préposition : rue Saint Jacques » (K. Togeby 1982 : 156).

En fait, dans le cas des noms de personne précédés d'un titre, l'usage est plus fluctuant que ne le dit K. Togeby. Par exemple, pour Général une seule rue sur 26 n'a pas de préposition intérieure (rue Général Guilhem); pour Commandant quatre noms de rues de Paris présentent un de intérieur, et quatre autres une jonction directe (rue Commandant Lamy, rue du Commandant Léandri) ; pour Docteur, on trouve $13 d u$ intérieur, contre 17 sans $d u$, (rue Docteur Potain, rue du Docteur Roux). Par ailleurs, on trouve aussi des noms de lieu en construction directe dans l'index alphabétique mais avec de sur le plan : boulevard (de) Sébastopol. Enfin, il arrive fréquemment que tel ou tel usager ajoute ou supprime un de intérieur.

31 Il n'en demeure pas moins une tendance statistiquement dominante à lier par de un Npr de lieu et à juxtaposer directement un Npr de personne. Cette bipartition est d'autant plus curieuse que le français aurait pu généraliser une seule construction pour les noms propres comme l'ont fait plusieurs autres langues. Ainsi en polonais, le génitif est de règle (Ul. Aleksandry), en latin c'est l'adjectif éponyme du constructeur (via Appia, commencée par Appius), en italien et en espagnol, c'est la construction directe, sauf après place ${ }^{10}$. 
Quelles sont donc les propriétés de ces deux structures qui ont entraîné leur spécialisation?

\subsection{Comparaison des odonymes de type rue de Rennes et rue Descartes}

Ces deux types d'odonymes diffèrent, à première vue, par le mode de jonction de leurs deux membres. Alors que de marque clairement une relation syntaxique faisant dépendre le $\mathrm{Npr} \mathrm{du} \mathrm{N} 1_{c}$, il est difficile de se prononcer sur le type de rapport qui unit N1c à N2pr dans les constructions directes. La plupart des grammaires l'identifient à une apposition, fonction grammaticale tardivement issue de la rhétorique et définie selon des critères hétérogènes ${ }^{11}$. Pour sa part, Noailly (1990) propose d'analyser comme un substantif épithète le $\mathrm{N} 2_{\mathrm{pr}}$ qui, dans les constructions en le $\mathrm{N} 1 \mathrm{~N} 2{ }_{\mathrm{pr}}$, sert à identifier le N1. Car, précise-t-elle, « le nom commun initial livre la classe dans laquelle se range N2. On a donc le nom de la classe, puis le nom d'une unité particulière dans cette classe »(p.135). Une telle analyse, qui se situe au niveau des structures grammaticales, ne rencontre même pas la question de la double facture des noms de rue à $\mathrm{N}_{\mathrm{pr}}$. Celle-ci ne se pose en effet que dans le champ de la signalétique des voies urbaines. Aussi est-il nécessaire de prendre en compte l'interaction de facteurs linguistiques généraux et de contraintes pragmatiques locales pour tenter d'expliquer pourquoi les odonymes combinant un N1c à un N2 se construisent selon deux patrons distincts.

Comparons rue Descartes à rue de Descartes. La construction en de pose un lien de détermination syntaxique qui invite à chercher une relation sémantique entre rue et Descartes, que le sens de la préposition de ne permet pas de spécifier. Mais c'est au niveau des référents désignés par rue et Descartes que l'on cherche à établir un rapport. On pense alors à une rue où Descartes a pu naître, habiter ou mourir. C'est-à-dire une rue qui partage la célébrité d'un grand philosophe. Or c'est précisément cette mise en rapport sémantique que brise la construction directe en proposant une structure binaire "hors syntaxe", définie par deux positions contiguës et ordonnées. Quand on juxtapose un chiffre ou une lettre à un nom catégorisateur: Nationale 10, Allée A, on distingue la position initiale de classificateur nominal de celle, finale, de spécificateur. Les deux positions sont occupées par deux ensembles finis spécifiques : le répertoire des catégories de rue pour la première et la suite des chiffres ou des lettres d'un alphabet pour la seconde. Il nous semble que les noms propres de personne en construction directe sont assimilables fonctionnellement aux chiffres et aux lettres, dépourvus de toute signification référentielle et n'ayant qu'une valeur différenciatrice au sein du paradigme dont ils relèvent ${ }^{12}$. Autrement dit, la référence à l'individu Descartes n'est pas pertinente au niveau de la structure dénominative $e^{13}$. Mais c'est en amont de la dénomination, au fondement du système honorifique même qu'elle trouve sa raison d'être. L'idée de Sully fut, comme le dit D. Milo (1986:287) « d'adopter des noms qui n'eussent pas de rapport direct avec le lieu auquel ils étaient imposés ». L'acte commémoratif consiste précisément dans le rappel du nom d'un individu et sert accessoirement de système différenciateur pour la nomenclature des rues. Et corollairement, la construction déterminante implique une caractérisation référentielle locale. La rue de l'Hôpital suppose qu'il y a (ou qu'il y a eu, au moment de la nomination) un hôpital dans ladite rue. 

rues trois étapes. La plus ancienne remonte à des habitudes médievales de désigner les rues, entre autres, d'après des repères géographiques familiers. Il y a donc une motivation référentielle à la base de ces dénominations, dont l'interprétation est compositionnelle, comme dans le cas précédemment évoqué de la rue de Paris. On rattachera à ce type de formation, les dénominations de rues qui se trouvent dans des quartiers (souvent aux environs des gares) où se regroupent des provinciaux venus de Bretagne par exemple. La seconde étape se situe à l'époque napoléonienne. Elle se caractérise par la multiplication des noms de lieu qui commémorent des batailles : avenue d'Iéna, rue d'Arcole, etc. Ces noms conservent en partie leur valeur référentielle locative, dans la mesure où ils évoquent métonymiquement une bataille à partir de l'endroit où elle "a eu lieu". Enfin, ce n'est qu'à une époque très récente que des noms de lieu étrangers, sans attaches référentielles, comme avenue de New York,ont été introduits dans la nomenclature des rues. Leur nombre encore infime et leur usage tardif attestent $a$ contrario la forte prédominance d'une interprétation référentielle pour les Npr de lieu. Interprétation qui a sans doute contribué à préserver la construction en de sur laquelle elle repose.

Certes l'optimisation sémiotique tendrait à imposer un schéma unique. Mais nous savons que l'histoire a fait de cette signalétique un ensemble hétérogène comportant différentes strates. Le système de classification en de préexiste donc au système plus récent qui comporte des noms propres de personnes. D'un côté, le système de classification ancien maintient l'expression d'une motivation sémantique topographique, de l'autre le système de classification moderne utilisant les noms propres de personnes ( $\left.\mathrm{N} 2{ }_{\text {pr-pers }}\right)$ n'exprime aucune espèce de motivation sémantique de ce type. Quelle peut donc être dans ces conditions la nature sémantique du rapport établi par le couplage syntagmatique du premier formant catégoriel $\mathrm{N} 1_{\mathrm{c}}$ et du second formant $\mathrm{N} 2_{\text {pr-pers }}$ ?

Si la mention du N2 ${ }_{\text {pr-pers }}$ n'est pas associée dans ce genre de couplage à l'expression d'une relation entre les référents respectivement désignés par $\mathrm{N} 1_{c}$ et $\mathrm{N} 2_{\text {pr-pers }}$, on peut en déduire que la relation établie par le couplage syntagmatique des deux formants est la solution minimale, fondée sur une simple relation d'ordre séquentiel, pour donner une forme linguistique à des dénominations complexes produites par une combinatoire taxinomique autonome. Au premier formant $\mathrm{N} 1_{\mathrm{c}}$ relativement constant (rue par exemple), correspond une variation du deuxième formant $\mathrm{N} 2{ }_{\text {pr-pers }}$ : Bonaparte / Gambetta, par exemple, corrélative à l'opposition rue Bonaparte et rue Gambetta, c'est-à-dire "la rue qui s'appelle rue Bonaparte" et "la rue qui s'appelle rue Gambetta". Faute d'une autre relation disponible, la relation d'appellation est dans ce cas la relation reconnue et chaque dénomination complexe ainsi constituée s'oppose à toutes les autres comme étant la seule à pouvoir identifier telle voie et non telle autre. D'un autre côté, contrairement à rue 
Bonaparte qui signifie exclusivement qu'il s'agit du nom d'une voie urbaine par opposition à d'autres noms possibles, rue de Paris peut signifier non seulement "la rue qui s'appelle rue de Paris" mais également "la rue qui va à Paris. Ainsi la préposition de trouve-t-elle ici un emploi sémantico-syntaxique qu'elle ne pourrait remplir dans l'autre système de dénomination où seule compte une structure binaire de type à la fois classificatoire et identifiante. Cet antagonisme assure le développement parallèle des deux schémas de noms de rue caractéristiques du français contemporain.

\section{Conclusion}

$\mathrm{Au}$ terme de cette promenade dans l'espace singulier des voies de circulation urbaine, on est obligé de constater que la belle ordonnance administrative des plaques de rues ne peut montrer que ce qu'elle a : une unité de façade. La double construction en $\mathrm{N} 1_{\mathrm{c}} \mathrm{de} N \mathrm{~N}_{\mathrm{pr}}$ ou N1 ${ }_{c} \mathrm{~N}_{\mathrm{pr}}$ témoigne de l'historicité de ce type de nomenclature. L'introduction de noms de personnes dans la signalétique ancienne a déplacé sensiblement le schème qui présidait à la nomination et à l'identification des voies. L'absence de préposition entre le terme catégoriel $\left(\mathrm{N}_{\mathrm{c}}\right)$ et le nom de personne $\left(\mathrm{N} 2_{\text {pr-pers }}\right.$ correspond en effet à une absence de relation sémantico-référentielle entre chacun de ces formants du nom de rue. C'est ainsi qu'on est passé d'une signalétique sémantiquement motivée par des critères topographiques à une combinatoire fondamentalement plus abstraite, dans laquelle les noms de personnes empruntés valent d'abord comme des éléments différenciateurs à l'intérieur de classes désignées par un stock relativement stable de termes catégoriels (rue , avenue, impasse...). Quoique différent de l'onomastique des voies de circulation non urbaines qui exigerait une étude spécifique, cette signalétique s'en approche néanmoins quelque peu puisque le couplage sans ligature des deux formants $\mathrm{N} 1_{c}$ et $\mathrm{N} 2_{\text {pr-pers }}$ suffit à constituer des formes dénommantes à structure binaire selon un mode classificatoire tout à fait analogue au système alphanumérique des noms de routes. Ce qui domine en effet dans de tels dispositifs d'identification, c'est la prégnance de la relation dénominative au détriment des autres relations sémantico-référentielles.

\section{BIBLIOGRAPHIE}

\section{Références}

ANDRIEUX-REIX N. (1996), « Séquences binominales non-prépositionnelles et relation dite “d'appartenance" en ancien français », Faits de langue, 7, La Relation d'appartenance.

BOSREDON B. (1997), Les titres de tableaux. Une pragmatique de l'identification, Paris, PUF.

BOSREDON B. (1998), « Les signalétiques de nomination ou quand le discours se fige », Rencontres Linguistiques Méditerranéennes, Tunis, CERES.

KLEIBER G. (1985), « Le projet Delors, la camarade Catherine », L'information grammaticale, 27.

MILO D. (1986), « Le nom des rues », Les lieux de mémoire T. II, éditeur P. Nora, Paris, Gallimard. 
NEVEU F. (1996), « La notion d'apposition en linguistique française : perspective historique », Le français moderne, LXIV, 1.

NEVEU F. (1998), Etudes sur l'apposition, Paris, Champion.

NOAILLY M. (1990), Le substantif épithète, Paris, PUF.

PALM L. (1989), « "On va à la Mouff" Etude sur la syntaxe des noms de rues en français contemporain », Acta Universitatis Upsaliensis, Studia Romanica Upsaliensia 45, Uppsala.

PEREC G. (1974), Espèces d'espaces, Paris, Galilée.

PETIT G. (1998), « Remarques sur la structuration sémiotique des locutions familières », Le figement lexical, Rencontres linguistiques Méditerranéennes, Tunis, CERES.

RASTIER F. (1991), « Peut-on définir sémantiquement le prototype ? », Sémiotiques I,1.

RIOUL R. (1983), « Les appositions dans la grammaire française », L'information grammaticale, 18.

TAMBA I. (à par.), « L'apposition, une étrange position syntaxique », Conférence à Séoul, le $1 / 12 / 1998$.

TAMBA I. (à par.), « Métaphore binominale et référence », Conférence à Séoul, le 5/12/1998.

TASSY P. édit. (1986), L'ordre et la diversité du vivant, Paris, Fayard/Fondation Diderot.

TOGEBY K. (1982), Grammaire française. Pub. par M. Berg, G.Merad et E. Spang-Hanssen. vol. I : Le nom, Copenhague, Akademisk forlag.

\section{Documentation}

Guide indicateur des rues de Paris dit Paris éclair, A. Leconte éditeur, rue Sainte-Croix-de-laBretonnerie.

Cartes IGN, cartes Michelin.

\section{NOTES}

1. Cf. D Milo (1986: 283) qui se demande «Comment ne pas être sensible à l'ingéniosité des révolutionnaires rémois qui, de la rue Tire-Vit, fait la rue de Foi-Conjugale (...) ; ou à celle du conseil municipal de Saint Etienne qui rebaptise la rue Saint-Honoré, Honoré de Balzac en 1905, puis Balzac tout court en 1907 ».

2. A ne pas confondre ici avec le terme de signalétique qui renvoie à la signalisation routière.

3. Lars Palm, dans une étude de 1989, que nous remercions M. Noailly de nous avoir indiquée et prêtée, recense à partir d'un corpus de 635 exemples de sources diverses, les 16 catégories suivantes : "allée, avenue, boulevard, carrefour, chaussée, cours, impasse, passage, place, pont, porte, promenade, quai, route, rue, square». Il faudrait ajouter: carré, hameau (hameau Boulainvilliers dans le 16e arrondissement à Paris), passerelle, ruelle, sente, sentier, également rencontrés dans certains répertoires.

4. $C f$. le guide indicateur des rues de Paris dit Paris éclair contenant une Nomenclature des rues de Paris.

5. Rappelons que nous nous limitons aux odonymes urbains français actuels, étant donné la diversité des signalétiques des voies urbaines selon les époques et les pays. On trouve par exemple une numérotation des voies urbaines à New-York (5ème avenue) ou dans certains quartiers de Beyrouth (rue 11) etc..

6. La préposition à est très rarement employée, comme dans quai aux Fleurs. 
7. Il arrive que la forme bisegmentale disparaisse comme dans Sébasto (pour boulevard Sébastopol), La Mouff' (pour rue Mouffetard). On considèrera qu'il s'agit là de cas de surnomination. Le registre de langue familier invite à cette interprétation. Par ailleurs, dans ces cas de surnomination, on tend à interpréter ces formes plutôt comme des noms de quartiers, de secteurs, éventuellement de carrefours (Cf. Ménilmuch, Nation, Etoile...). Le caractère bisegmental apparaît donc comme un marqueur obligatoire dans la signalétique des noms de rues.

8. Nous citons ce passage d'après L. Palm (1989: 9).

9. Certaines cartes touristiques ainsi que les panneaux de signalisation utilisent ces dénominations avec article ou sans article défini, avec majuscule ou sans majuscule sur l'article ; l'usage semble à cet égard très flottant.

10. Merci à G. Serbat qui nous a signalé les constructions du russe et du latin et à J-C Anscombre et L. Greco à qui nous devons nos informations pour l'espagnol et l'italien.

11. R. Rioul rappelle la complexité de l'apposition qui, selon la nomenclature grammaticale de 1975, réunit des faits linguistiques aussi différents que le lait fraise ou la rue Victor Hugo (R. Rioul, 1983 : 21). Et F. Neveu (1996, 1998), en retraçant l'histoire de l'apposition, montre de quel compromis rhétorico-grammatical elle résulte.

12. On rapprochera ce type de chaînage binaire à fonction à la fois classificatoire (une première place pour un nom de classe) et individualisante (une deuxième place pour une marque d'individu) de ces formes de chaînages plus complexes étudiées par G. Petit (Petit G. 1998) dans les locutions familières (comme par exemple «manger les pissenlits par la racine »), suites dans lesquelles le chaînage est avant tout une structure sémantique.

13. On ne peut faire correspondre à ce type de construction binaire définie par deux positions contiguës et ordonnées aucune autre relation sémantico-syntaxique que celle qui fait de l'ensemble de la structure un bloc dénommant du type: le N1 appelé N1N2 pr. C'est ce qui différencie ces dénominations complexes des "compositions nominales non lexicalisées " comme le projet Delors ou l'année Victor Hugo étudiées par G. Kleiber (1985: 5), dans lesquelles le Npr renvoie effectivement à l'individu porteur du nom propre.

14. Comme nous l'a signalé $F$. Renaud, pour les désignations familières des routes, le spécificateur courant est celui de la localité que l'on veut atteindre. Ainsi appellera-t-on la même route route de Boutiers ou route de Cognac, selon que l'on se déplace de Cognac à Boutiers ou inversement. De même certaines portes de Paris, comme Porte de Versailles, sont nommées d'après la destination visée à partir de Paris.

\section{RÉSUMÉS}

Cet article montre que les noms de rue se construisent aujourd'hui en français selon deux patrons réguliers, couplant un premier nom commun à fonction catégorisante, tel que rue, avenue, etc., à un second nom propre à fonction individualisante. Mais dans le type rue Descartes, le nom propre est un nom de personne qui se postpose directement au nom catégoriel, tandis que le type rue de Rennes présente un nom de lieu joint par la préposition de. Ces structures binaires constituent une appellation unique, partageant le fonctionnement dénominatif des systèmes alphanumériques qu'utilise la cartographie routière. Seule l'interprétation référentielle est concernée par leur différence de facture. Le nom propre locatif est sémantiquement motivé dans la mesure où il implique une certaine relation avec le lieu dont il porte le nom, alors que le nom 
propre de personne n'entretient qu'un rapport conventionnel de commémoration avec l'individu éponyme.

Contemporary french street names are built in two regular patterns: a common noun indicating the type of street (rue, avenue, etc.) is followed by a proper noun making the name individual. In one pattern (rue Descartes), the proper noun names a person and follows the common noun directly. In the second (rue de Rennes), the proper noun refers to a place and is coupled to the common noun by the preposition de. These two-noun structures assign a unique name to each street, just as names composed of a common noun followed by an alphanumeric code assign unique names to roads on road maps. The difference between the two patterns solely lies in their referential basis. In street names, the reference to places is semantic with some underflying relation between the streets and the corresponding places, whereas persons are only named conventionally to recall their memory.

\section{AUTEURS \\ BERNARD BOSREDON}

Paris 3

IRÈNE TAMBA

ehess 